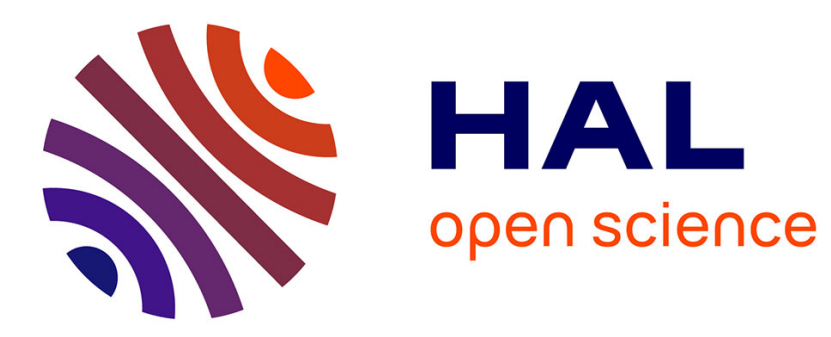

\title{
Slip Length Measurement of Confined Air Flow on Three Smooth Surfaces
}

Yunlu Pan, Bharat Bhushan, Abdelhamid Maali

\section{To cite this version:}

Yunlu Pan, Bharat Bhushan, Abdelhamid Maali. Slip Length Measurement of Confined Air Flow on

Three Smooth Surfaces. Langmuir, 2013, 29 (13), pp.4298-4302. 10.1021/la400199k . hal-00834329

\section{HAL Id: hal-00834329 \\ https://hal.science/hal-00834329}

Submitted on 9 Mar 2018

HAL is a multi-disciplinary open access archive for the deposit and dissemination of scientific research documents, whether they are published or not. The documents may come from teaching and research institutions in France or abroad, or from public or private research centers.
L'archive ouverte pluridisciplinaire HAL, est destinée au dépôt et à la diffusion de documents scientifiques de niveau recherche, publiés ou non, émanant des établissements d'enseignement et de recherche français ou étrangers, des laboratoires publics ou privés.

\section{(1) $\$(0)$}

Distributed under a Creative Commons Attribution - NonCommercial - ShareAlikel 4.0 


\title{
Slip Length Measurement of Confined Air Flow on Three Smooth Surfaces
}

\author{
Yunlu Pan, ${ }^{\dagger, \ddagger}$ Bharat Bhushan, ${ }^{* \dagger}$ and Abdelhamid Maali ${ }^{\S}$ \\ ${ }^{\dagger}$ Nanoprobe Laboratory for Bio and Nanotechnology \& Biomimetics (NLB ${ }^{2}$ ), The Ohio State University, 201 W. 19th Avenue, \\ Columbus, Ohio 43210 1142, United States \\ ${ }^{\ddagger}$ School of Mechatronics Engineering, Harbin Institute of Technology, Harbin 150001, PR China \\ ${ }^{\S}$ Laboratoire Ondes et Matière d'Aquitaine (LOMA), Université Bordeaux I351 cours de la Liberation, F 33405 Talence, France
}

\begin{abstract}
An experimental measurement of the slip length of air flow close to three different solid surfaces is presented. The substrate was driven by a nanopositioner moving toward an oscillating glass sphere glued to an atomic force microscopy (AFM) cantilever. A large separation distance was used to get more effective data. The slip length value was obtained by analyzing the amplitude and phase data of the cantilever. The measurements show that the slip length does not depend on the oscillation amplitude of the cantilever. Because of the small difference among the slip lengths of the three surfaces, a simplified analysis method was used. The results show that on glass, graphite, and mica surfaces the slip lengths are 98, 234, and $110 \mathrm{~nm}$, respectively.

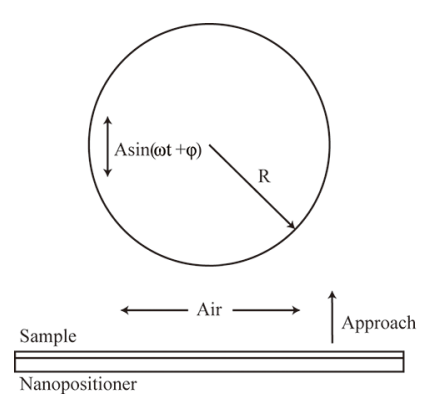

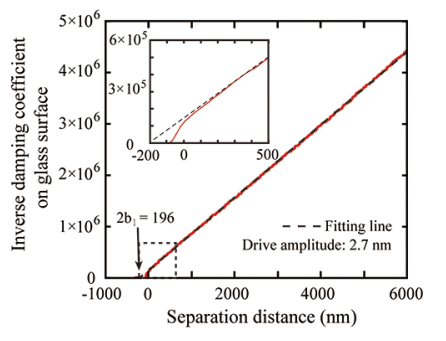

\section{INTRODUCTION}

The boundary condition of fluid flow has been of interest since the 19th century. ${ }^{1-7}$ The slip condition, which means the relative velocity of the fluid close to the surface, is not equal to zero and can reduce drag during fluid flow. ${ }^{8,9}$ Slip length $b$ is used to present the slip condition. The tangential velocity of fluid flow under the slip condition is ${ }^{1}$

$$
v=\left.b \frac{\mathrm{d} v}{\mathrm{~d} z}\right|_{z=0}
$$

where $\nu$ is the tangential velocity and $z$ is the axis perpendicular to the wall.

The existence of slip was first predicted by Maxwell ${ }^{2}$ for gas flow close to a wall. Slip is important in ultrathin films of fluids. For nanoelectromechanical systems (NEMS), the available space for the gas is substantially reduced, and the large surface to volume ratio for these devices enables the boundary flow to play an important role. The boundary slip is expected to reduce the drag of the system because it reduces the friction of the gas flow on the surfaces of the devices.

The slip condition of air flow has been studied by several groups using atomic force microscopy.,10-14 Unlike the measurement of the slip length of a liquid, ${ }^{9,15-17}$ dynamic methods were mostly chosen because of the low viscosity of air. The high quality factor of the cantilevers in air makes any variation in the damping accurately measurable. The slip length value is extracted from the measurements of the damping variation versus the thickness of the confined air. The measured slip length can also be used to determine the so called accommodation coefficient that characterizes the collision between the gas molecules and the solid surface. The accommodation coefficient characterizes the value of the fraction of gas molecules reflected diffusively from the surface. Specifically, the accommodation coefficient is equal to 1 for a pure diffuse reflection and 0 for a pure specular reflection. The accommodation coefficient depends on the surface nature and the gas composition and also on the temperature.

Maali and Bhushan ${ }^{7}$ used an acoustically driven oscillation of a sphere glued to an AFM cantilever to measure the damping versus the distance. They calculated a slip length of $118 \pm 10$ $\mathrm{nm}$ on the glass surface. Siria et al. ${ }^{10}$ analyzed the thermally driven oscillation of a cantilever as it gradually approaches a wall in parallel geometry and hypothesized the perfect slip boundary conditions for the air flow on their surfaces. More recently, Honig et al. ${ }^{12}$ and Bowles and Ducker ${ }^{14}$ used similar techniques (thermal excitation) and reported a finite slip in agreement with the results of Maali and Bhushan ${ }^{7}$ with a slip value ranging from 100 to $630 \mathrm{~nm}$ depending on the nature of the surfaces and their preparation.

The aims of this paper are the following: (1) To demonstrate that the damping versus the separation is independent of the amplitude of vibration and thus the extracted slip length is not linked to the amplitude value. (2) To probe the damping variation in a large range of separation between the sphere and the solid surface in order to simplify the extraction of the slip length. This approach allows the extraction of the slip length and the accommodation coefficient of air flow on three different surfaces. 


\section{MODELING}

For a cantilever oscillating with a small amplitude, the interaction force acting on the cantilever can be linearized and has two contributions; one is a conservative term $\left(-k_{\mathrm{H}} z\right)$ resulting from the elastic compression of the gas and the other is a dissipative term $\left(-\gamma_{\mathrm{H}} \dot{z}\right)$ resulting from viscous lubrication damping during the squeezing of the gas. The motion of the cantilever is then described by

$$
m^{*} \ddot{z}+\left(\gamma_{0}+\gamma_{\mathrm{H}}\right) \dot{z}+\left(k_{1}+k_{\mathrm{H}}\right) z=F_{\mathrm{D}} \exp (j \omega t)
$$

where $m^{*}$ is the effective mass of the cantilever, $z$ is the instantaneous position of the cantilever along the vertical axis, $k_{1}$ is the cantilever stiffness, $k_{\mathrm{H}}$ is the hydrodynamic interaction stiffness, $F_{\mathrm{D}}$ is the driving force $\left(F_{\mathrm{D}}=k_{1} A_{0} / Q_{0}\right), A_{0}$ is the amplitude of the oscillation far from the surface, $Q_{0}$ is the quality factor far from the surface, and $\omega$ is the driving frequency.

$\gamma_{\mathrm{T}}$ is the total damping, which is the sum of the bulk viscous damping $\gamma_{0}$ and the hydrodynamic lubrication damping $\gamma_{\mathrm{H}}$ that depends on the separation between the sphere and the solid surface. The effective mass is related to the cantilever stiffness by the equation $\omega_{0}=\left(k_{1} / m^{*}\right)^{1 / 2}$

The steady state solution $z=A \exp j(\omega t+\varphi)$ of eq 2 gives the total damping value

$$
\gamma_{\mathrm{T}}=\frac{k_{\mathrm{l}} A_{0}}{Q_{0} \omega A} \sin (\varphi)
$$

where $A$ and $\varphi$ are the amplitude and phase of the oscillating tip.

For a sphere moving toward a surface in fluid, under the nonslip condition, the hydrodynamic lubrication force acting on the sphere can be given by the Taylor equation

$$
F_{\mathrm{H}}=\frac{6 \pi \mu R^{2}}{D} \frac{\mathrm{d} z}{\mathrm{~d} t}
$$

where $R$ is the radius of the sphere, $\mu$ is the viscosity of the air, and $D$ is the distance of the sphere from the surface. According to Vinogradova, ${ }^{18}$ under the boundary slip condition the hydrodynamic force $F_{\mathrm{H}}$ can be written as

$$
F_{\mathrm{H}}=\gamma_{\mathrm{H}} \frac{\mathrm{d} z}{\mathrm{~d} t}=f^{*} \frac{6 \pi \mu R^{2}}{D} \frac{\mathrm{d} z}{\mathrm{~d} t}
$$

if both surfaces have the same slip length $b$,

$$
f^{*}=\frac{2 D}{6 b}\left[\left(1+\frac{D}{6 b}\right) \ln \left(1+\frac{6 b}{D}\right)-1\right]
$$

If the two surfaces have different slip lengths $b_{1}=b$ and $b_{2}=(1$ $+k) b$, then

$$
\left\{\begin{array}{c}
f^{*}=-\frac{A D}{B C}-\frac{2 D}{C-B}\left[\frac{(B+D)(B-A)}{B^{2}} \ln \left(1+\frac{B}{D}\right)\right. \\
\left.-\frac{(C+D)(C-A)}{C^{2}} \ln \left(1+\frac{C}{D}\right)\right] \\
A=b(2+k) \\
B=2 b\left(2+k+\sqrt{1+k+k^{2}}\right) \\
C=2 b\left(2+k-\sqrt{1+k+k^{2}}\right)
\end{array}\right.
$$

When both surfaces have the same slip length, at a large separation distance $D \gg b, f^{*}$ can be expanded in series to first order:

$$
f^{*} \approx 1-\frac{2 b}{D}
$$

Therefore, at large separation distances the hydrodynamic force is

$$
F_{\mathrm{H}}=\gamma_{\mathrm{H}} \frac{\mathrm{d} z}{\mathrm{~d} t} \approx \frac{6 \pi \mu R^{2}}{D} \frac{\mathrm{d} z}{\mathrm{~d} t}\left(1-\frac{2 b}{D}\right) \approx \frac{6 \pi \mu R^{2}}{D+2 b} \frac{\mathrm{d} z}{\mathrm{~d} t}
$$

Then we have

$$
\frac{1}{\gamma_{\mathrm{H}}} \approx \frac{(D+2 b)}{6 \pi \mu R^{2}}
$$

The value of the slip length can be obtained by fitting the curve of the inverse of the damping to eq 8 . The advantage of using the data of the inverse damping instead of the damping is that the final result of the slip length appears as a shift distance of the curves that is not sensitive to the value of the cantilever stiffness and other parameters such as the radius of the sphere and the viscosity of air.

When both surfaces have different slip lengths, $f^{*}$ is controlled by the sum of the slip lengths $b_{\text {total }}=b_{1}+b_{2} .{ }^{14}$ If the difference between $b_{1}$ and $b_{2}$ is not too large, then $f^{*}$ is similar to the case of equal slip length. Therefore, at large separation distances $f^{*} \approx 1-\left(b_{\text {total }} / D\right)$ and

$$
\frac{1}{\gamma_{\mathrm{H}}} \approx \frac{\left(D+b_{\text {total }}\right)}{6 \pi \mu R^{2}}
$$

$b_{\text {total }}=b_{1}+b_{2}$ can be obtained by fitting the curve of the inverse damping. During our experiments, the slip length of the glass sphere on the tip should be measured first by oscillating toward a glass surface that has the same slip length. Then the glass sphere can be used to measure the slip length of other surfaces.

\section{EXPERIMENTAL SETUP}

The experiment was performed using an AFM (Dimension 3000) with a Nanoscope IV controller (Bruker Instruments). Because of the small viscosity of air, the hydrodynamic force is relatively small. To maximize the hydrodynamic force, a large spherical glass particle with a diameter of $134 \mu \mathrm{m}$ was used. Meanwhile, the hydrodynamic force due to the cantilever is too small to compare with the hydrodynamic force due to the particle and can be neglected because the distance between the surface and the cantilever is much larger (larger than $134 \mu \mathrm{m}$ ). The particle was glued to the end of a rectangular cantilever (LRCH $225 \mathrm{C} 3 / \mathrm{R}$, Team Nanotec $\mathrm{GmbH}$ ) using an epoxy glue (Araldite, Bostik, Coubert). The amplitude and phase of the tip were measured using a lock in amplifier (Signal Recovery model 7280), and the tip was also driven to oscillate by the lock in amplifier. During the experiment, the tip's position was fixed, and the sample was driven to approach and separate from the tip by a nanopositioner (NPZ300M, nPoint Inc.) that allows a large displacement (up to $300 \mu \mathrm{m}$ with a drive voltage of $20 \mathrm{~V}$ ) with high accuracy under closed loop control (Figure 1). To drive the nanopositioner, a signal generator (33120A, $\mathrm{HP}$ ) was used to generate a triangular wave with a frequency of 0.02 $\mathrm{Hz}$ and a peak to peak value of $4 \mathrm{~V}(60 \mu \mathrm{m}$ for the nanopositioner). The deflection signal of the tip was also recorded by the lock in amplifier from the deflection signal we know when the sphere touches the surfaces, and we get the 0 point of the separation distance.

Three smooth surfaces were used: glass, mica, and graphite. The mica and graphite surfaces are atomically smooth surfaces. The glass surface and the glass sphere have roughnesses of 1.3 and $2 \mathrm{~nm}$, respectively, measured over a $1 \mu \mathrm{m}^{2}$ area. It is assumed that the slip lengths on the glass surface and on the glass sphere are the same. The slip length on the glass surface was measured first. Then the slip lengths on the mica and the graphite were measured using a glass 


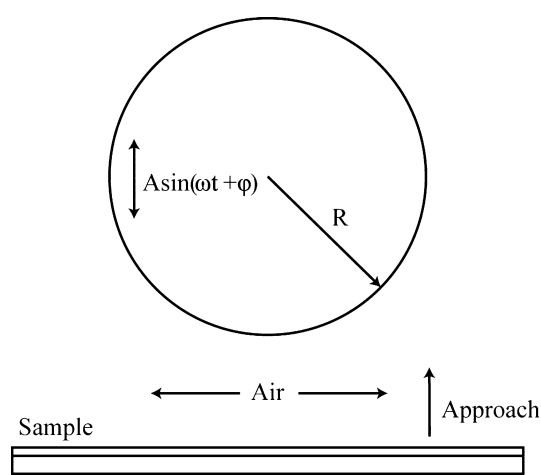

Nanopositioner

Figure 1. Schematic of a surface that is approaching an oscillating sphere with a very low velocity in air driven by a nanopositioner.

sphere with a known value of the slip length. The experiments were run at a fixed room temperature of $20^{\circ} \mathrm{C}$ with a humidity of $35-40 \%$.

The resonance frequency of the cantilever was measured to be 7.108 $\mathrm{kHz}$, as shown in Figure 2, and the quality factor was calculated to be 974. The stiffness of the cantilever can be measured using the noise method $^{19}$ as $2.4 \mathrm{~N} / \mathrm{m}$.

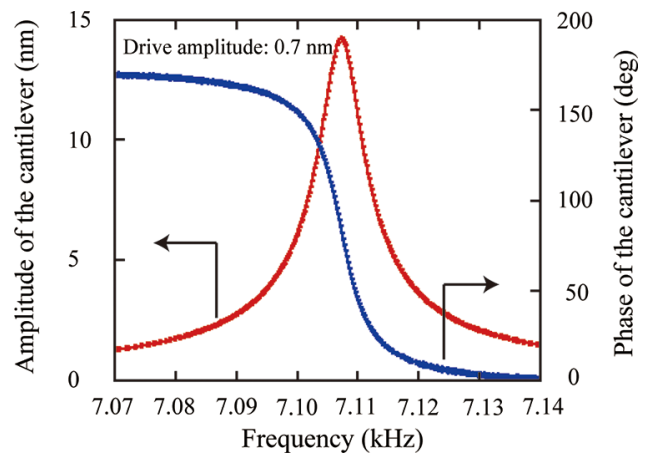

Figure 2. Amplitude and phase spectra of the cantilever with a sphere glued to the top.

\section{RESULTS AND DISCUSSION}

The amplitude, deflection, and phase of the cantilever measured on the mica surface are shown in Figure 3, and on the basis of the deflection plot, the hard contact position can be determined to set the 0 distance for all of the data including the amplitude and phase. By analyzing the data, the inverse damping coefficient on the glass surface can be obtained and is shown in Figure 4. The drive amplitude was $2.7 \mathrm{~nm}$. The total slip length $2 b_{1}$ can be obtained by fitting the plot at a distance of about 1000 to $6000 \mathrm{~nm}$ by eq 8 . Then the final slip length can be calculated to be $98 \pm 19 \mathrm{~nm}$. The uncertainty mainly comes from the different fitting range. The inverse damping coefficients measured on the mica and the graphite surfaces are shown in Figures 5 and 6 with drive amplitudes of 1.4 and $1.2 \mathrm{~nm}$, respectively. The curves of the inverse damping coefficient on the three surfaces have nearly the same slope, and the total slip on the mica and the graphite surfaces can be obtained by fitting the plot to eq 9 at the same range of distance as on the glass surface. Then the slip lengths on the mica and the graphite surfaces can be calculated with the measured value of the slip length on the glass surface as $110 \pm 21$ and $234 \pm 29$ $\mathrm{nm}$, respectively. On the basis of the measured values of the slip

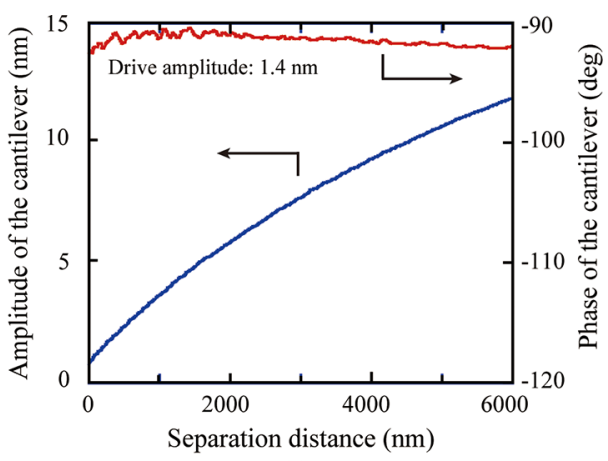

(a)

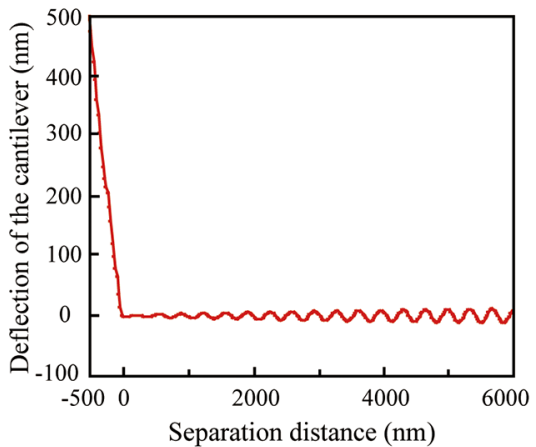

(b)

Figure 3. (a) Amplitude and phase of the cantilever measured on the mica surface. (b) Deflection of the cantilever measured as the glued sphere approaches the mica surface that allows one to determine the zero distance contact position of the two surfaces.

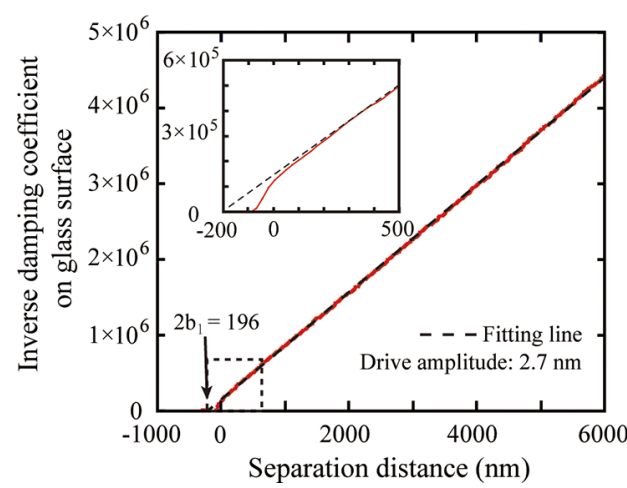

Figure 4. Inverse damping coefficient of the cantilever on a glass surface using eq 8 to fit the data and get the total slip length of $2 b_{1}=$ $196 \mathrm{~nm}$. The drive amplitude of the cantilever was $2.7 \mathrm{~nm}$.

length, the accommodation coefficients $\sigma$ of the three surfaces can be calculated by ${ }^{13,20}$

$$
b=\lambda\left(\frac{2.01}{\sigma}-0.73-0.16 \sigma\right)
$$

where $\lambda$ is the mean free path of the air with a value of $68 \mathrm{~nm} .{ }^{21}$ The measured slip lengths of air and the calculated accommodation coefficients of the three surfaces are shown in Table 1. Note here that for a given surface the accommodation coefficient depends on the composition of the gas and the temperature. The experiments described in this Article were conducted under the same conditions of temperature and relative humidity for all surfaces. A comparison of the results on different surfaces suggests that the slip length is sensitive to the roughness. The rough glass 


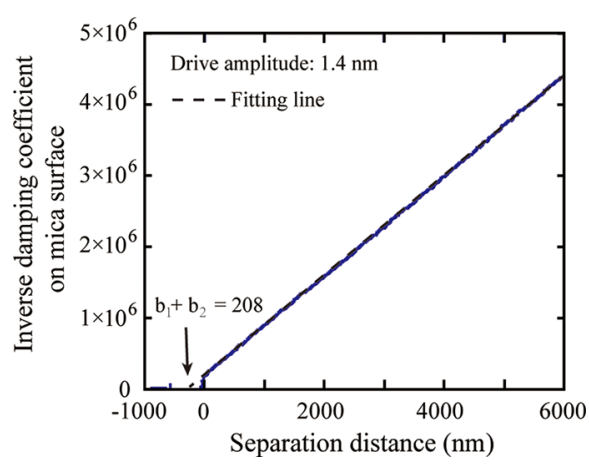

Figure 5. Inverse damping coefficient on the graphite surface using eq 9 to fit the data and get the total slip length of $b_{1}+b_{2}=208 \mathrm{~nm}$. The drive amplitude of the cantilever was $1.4 \mathrm{~nm}$.

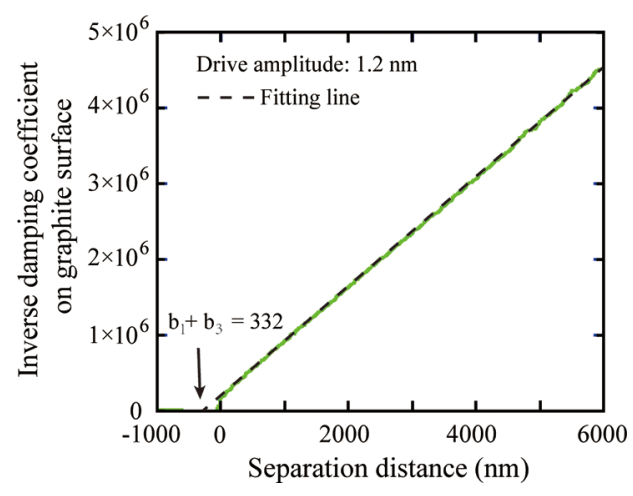

Figure 6. Inverse damping coefficient on the graphite surface using eq 9 to fit the data and get the total slip length of $b_{1}+b_{3}=332 \mathrm{~nm}$. The drive amplitude of the cantilever was $1.2 \mathrm{~nm}$.

Table 1. Measured Slip Length on Three Surfaces

\begin{tabular}{lcc} 
surface & measured slip length $(\mathrm{nm})$ & accommodation coefficients \\
glass & $98 \pm 19$ & $0.87 \pm 0.10$ \\
graphite & $234 \pm 29$ & $0.47 \pm 0.05$ \\
mica & $110 \pm 21$ & $0.81 \pm 0.11$ \\
\hline
\end{tabular}

surface produced a lower slip length and a greater accommodation of the gas as expected. The relative humidity can also affect the accommodation coefficient because the adsorbed water on the surfaces causes inelastic collision, allowing a greater accommodation coefficient (smaller slip length). The wettability of the graphite surface is lower than the mica and glass surfaces, and it may explain the higher slip length and the lower accommodation coefficient.

Figure 7 shows an example of the inverse of the damping coefficient with different driving amplitudes on the graphite surface. The data on the mica and the glass surfaces and for a given surface the inverse damping curves of different driving amplitudes coincide with each other. As a result, the measured value of the slip length on each surface did not obviously change by changing the drive amplitude. The slip lengths with $0.6,1.2$, and $2.4 \mathrm{~nm}$ driving amplitudes are $240 \pm 29,234 \pm 29$, and $249 \pm 31 \mathrm{~nm}$, respectively. Siria et al. ${ }^{10}$ hypothesized perfect slip based on the assumption that the boundary condition can be "strongly modified" by changing the driving amplitude of the cantilever. The experimental result supports an opposite result that the slip length of air is independent of the driving amplitude of the cantilever and is not infinite. Furthermore, our experiment also shows that for a given driving

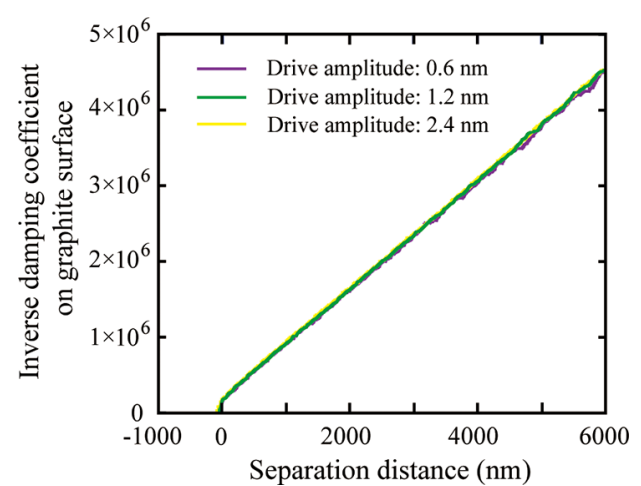

Figure 7. Inverse damping coefficient on a graphite surface with three different driving amplitudes: 0.6, 1.2, and $2.4 \mathrm{~nm}$.

amplitude the measured damping fits very well with the model that combines the Taylor equation with a slip function that assumes a constant slip length. If the slip length depends on the amplitude of vibration, then it should also depend on the distance because the amplitude depends on the distance, and thus the measurement should diverge from the theoretical model.

Compared to the work by Maali and Bhushan, ${ }^{7}$ thanks to the nanopositioner that can provide greater displacement, the effective separation distance for the fit is increased from 2000 to $6000 \mathrm{~nm}$. Equations 8 and 9 used for fitting are effective when $D \gg b$, which means the larger the separation distance, the better the fit. Also, a larger separation distance yields more information that can reduce the system uncertainty.

\section{CONCLUSIONS}

A dynamic AFM method with large separation distance for measuring the slip length is carried out. A nanopositioner was used to break the limitation of the AFM by giving a separation distance of up to $300 \mu \mathrm{m}$. Experiments were performed on glass, graphite, and mica with a maximum separation distance of $60 \mu \mathrm{m}$. The results show that there is a large amount of slip on all three surfaces: on the glass surface the slip length of air flow is $98 \pm 19 \mathrm{~nm}$, on the graphite surface the slip length of air flow is $234 \pm 29 \mathrm{~nm}$, and on the mica surface the slip length of air flow is $110 \pm 21 \mathrm{~nm}$. The accommodation coefficients were also calculated to be $0.87 \pm 10,0.47 \pm 0.05$, and $0.81 \pm 0.11$ on the glass, graphite, and mica surfaces, respectively. The slip lengths were measured with different driving amplitudes to obtain the same results that show the independence of boundary slip on the oscillation amplitude of the cantilever. The slip lengths with $0.6,1.2$, and $2.4 \mathrm{~nm}$ driving amplitudes are $240 \pm 29,234 \pm 29$, and $249 \pm 31 \mathrm{~nm}$, respectively.

\section{AUTHOR INFORMATION}

\section{Corresponding Author}

*E mail: bhushan.2@osu.edu.

Notes

The authors declare no competing financial interest.

\section{ACKNOWLEDGMENTS}

Y.P. acknowledges financial support from the Chinese Scholar ship Council. 


\section{REFERENCES}

(1) Navier, C. L. M. H. Memoire sur les lois du mouvement des fluides. Mem. Acad. Sci. Inst. Fr. 1823, 389-440.

(2) Maxwell, J. C. On the dynamical theory of gases. Trans. R. Soc. London 1867, 170, 231.

(3) Millikan, R. A. The general law of fall of a small spherical body through a gas, and its bearing upon the nature of molecular reflection from surfaces. Phys. Rev. 1923, 21, 217.

(4) Morris, D. L.; Hannon, L.; Garcia, A. L. Slip length in a dilute gas. Phys. Rev. A 1992, 46, 5279-5281.

(5) Bhushan, B. Tribology and Mechanics of Magnetic Storage Devices, 2nd ed.; Springer Verlag: New York, 1996.

(6) Bhushan, B. Springer Handbook of Nanotechnology, 3rd ed., Springer Verlag: Heidelberg, Germany, 2010.

(7) Maali, A.; Bhushan, B. Slip length measurement of confined air flow using dynamic atomic force microscopy. Phys. Rev. E 2008, 78, 027302.

(8) Ou, J.; Perot, B.; Rothstein, J. P. Laminar drag reduction in microchannels using ultrahydrophobic surfaces. Phys. Fluids 2004, 16, 4635-4643.

(9) Bhushan, B.; Wang, Y.; Maali, A. Boundary slip study on hydrophilic, hydrophobic, and superhydrophobic surfaces with dynamic atomic force microscopy. Langmuir 2009, 14, 8117-8121.

(10) Siria, A.; Drezet, A.; Marchi, F.; Comin, F.; Huant, S.; Chevrier, $\mathrm{J}$. Viscous cavity damping of a microlever in a simple fluid. Phys. Rev. Lett. 2009, 102, 254503.

(11) Drezet, A.; Siria, A.; Huant, S.; Chevrier, J. Giant slip lengths of a simple fluid at vibrating solid interfaces. Phys. Rev. E 2010, 81, 046315 .

(12) Honig, C. D. F.; Sader, J. E.; Mulvaney, P.; Ducker, W. A. Lubrication forces in air and accommodation coefficient measured by a thermal damping method using an atomic force microscope. Phys. Rev. E 2010, 81, 056305.

(13) Honig, C. D. F.; Ducker, W. A. Effect of molecularly thin films on lubrication forces and accommodation coefficients in air. J. Phys. Chem. C 2010, 114, 20114-20119.

(14) Bowles, A.; Ducker, W. Gas flow near a smooth plate. Phys. Rev. E 2011, 83, 056328.

(15) Bonaccurso, E.; Kappl, M.; Butt, H. J. Hydrodynamic force measurements: boundary slip of water on hydrophilic surfaces and electrokinetic effects. Phys. Rev. Lett. 2002, 88, 076103.

(16) Bonaccurso, E.; Butt, H. J.; Carig, V. S. J. Surface roughness and hydrodynamic boundary slip of a newtonian fluid in a completely wetting system. Phys. Rev. Lett. 2003, 90, 144501.

(17) Neto, C.; Evans, D. R.; Bonaccurso, E.; Butt, H. J. Boundary slip in Newtonian liquids: a review of experimental studies. Rep. Prog. Phys. 2005, 68, 2859.

(18) Vinogradova, O. I. Drainage of a thin liquid film confined between hydrophobic surfaces. Langmuir 1995, 11, 2213-2220.

(19) Matei, G. A.; Thoreson, E. J.; Pratt, J. R.; Newell, D. B.; Burnham, N. A. Precision and accuracy of thermal calibration of atomic force microscopy cantilevers. Rev. Sci. Instrum. 2006, 77, 083703.

(20) Lilley, C. R.; Sader, J. E. Velocity profile in the Knudsen layer according to the Boltzmann equation. Proc. R. Soc. London, Ser. A 2008, 464, 2015.

(21) Jennings, S. G. The mean free path in air. J. Aerosol Sci. 1988, 19, $159-166$. 